\title{
Optimization of Wireless Sensor Network Lifetime by Deploying Relay Sensors
}

\author{
B.Brahma Reddy \\ ECE, VBIT, Aushapur, Ghatkesar, RngaReddy district \\ Hyderabad, India-501301
}

\author{
K.Kishan Rao \\ Director, Vaagdevi College of Engineering \\ Bollikunta, Warangal, India- 506005
}

\begin{abstract}
Topology control in wireless sensor networks helps to lower node energy consumption by reducing transmission power and by confining interference, collisions and consequently retransmissions. Decrease in node energy consumption implies probability of increasing network lifetime. In this paper, firs popular topology control algorithms are used for analyzing optimizing the power consumption in the wireless sensor network and later proposed a novel technique wherein power consumption is traded with additional relay nodes. Later relay nodes are introduced to make the network connected without increasing the transmit power. The relay node decreases the transmit power required while it may increase end-to-end delay. This paper designs and analyzes an algorithm that place an almost minimum number of relay nodes required to make network connected. Greedy version of this algorithm is implemented and demonstrated in simulation that it produces a high quality link. InterAvg, InterMax (no of nodes that can offer interference) MinMax, and MinTotal are used as metrics to analyze and compare various algorithms. Matlab and NS-2 are used for simulation purpose.
\end{abstract}

\section{Key Words}

Energy saving, sensor networks, Interference, network connectivity, topology control

\section{INTRODUCTION}

Sensor network applications became popular due to their easy and rapid deployment processes. They can be deployed even into hazardous environments. These networks monitor outdoor environments and provide crucial data for emergency situations. Hence network connectivity is utmost important. They also work under extreme conditions such as noise and hostile atmosphere. They must work with minimum energy so that they work longer periods and offer minimal interference. Topology control can play major role in reducing node power consumption and extend network life time. In order to conserve the energy, the nodes are preferably configured at low transmit power. When the sensors are deployed at random locations, each node is to be configured at different transmit power levels making the network heterogeneous. However, it is possible to make the network homogenous by adding additional relays nodes at certain places which also conserve the network energy.

An efficient and energy conservation multi-hop wireless sensor network is discussed here. If the node are deployed at random places and all nodes are configured uniformly with low transmit power, obviously, there is high probability of forming an unconnected network as shown in figure1. Such unconnected network can be converted into connected network by i) adjusting transmit power of each node to appropriate level as shown in figure 2 ii) deploying relay nodes without changing transmit power.

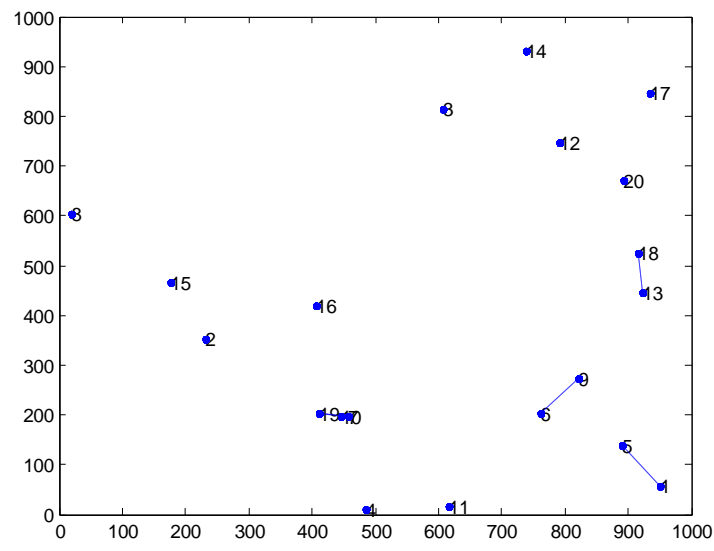

Figure.1 20-node unconnected network with uniform $\boldsymbol{P}_{t}=0.1$

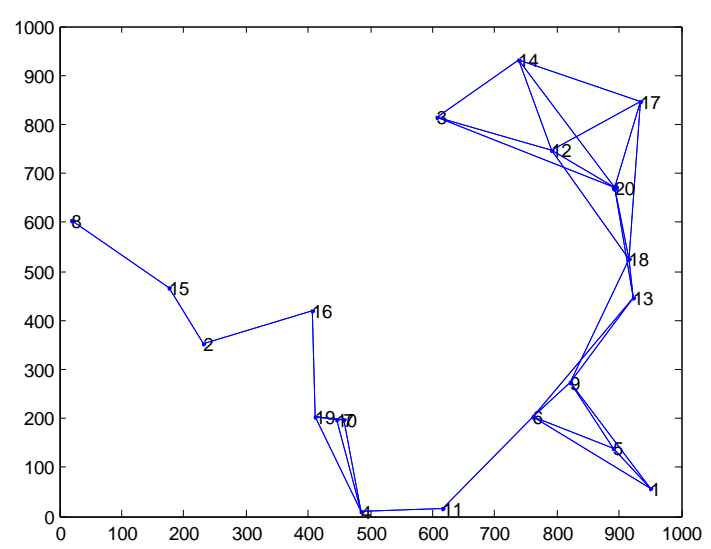

Figure2. 20-node connected network using non-uniform $\boldsymbol{P}_{\boldsymbol{t}}$

In the later case, there can be seen two types of nodes; i) original nodes which comprises of sensor and wireless transceiver ii) relay node which comprises of only wireless transceiver as shown in figure 3 .

Wireless communication link can be divided into multiple segments; and the segments can be connected through relay node; from following mathematical equations, it is known that transmit power required is less when the link is split into multiple segments. For simplicity, free space communication is assumed. 


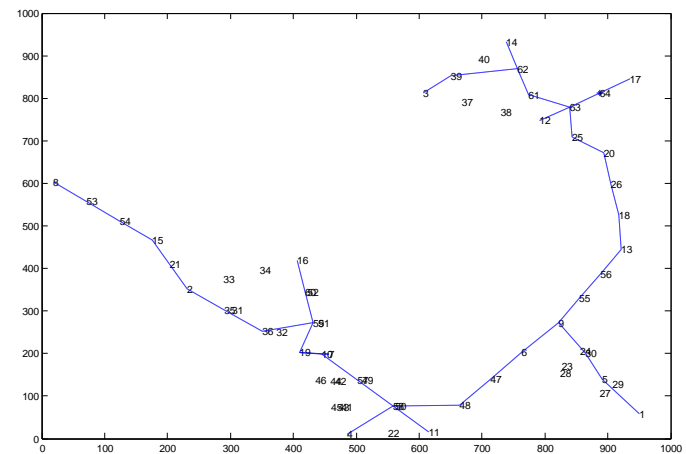

Figure.3 20-node connected network with $\boldsymbol{P}_{t}=\mathbf{0 . 1}$ after adding relay nodes

$\mathrm{T}$ is transmitting sensor node. $\mathrm{R}$ is receiving sensor node. $\mathrm{Y}$ is relay node. $\mathrm{D}$ is distance between $\mathrm{T} \& \mathrm{R}$. After adding relay node $\mathrm{Y}$, revised distance between $\mathrm{T} \& \mathrm{Y}$ and $\mathrm{Y} \& \mathrm{R}$ is $d / 2$. From the following equations, it can be seen that transmit power of figure $4 \mathrm{a}$ is more than transmit power of figure $4 \mathrm{~b}$.

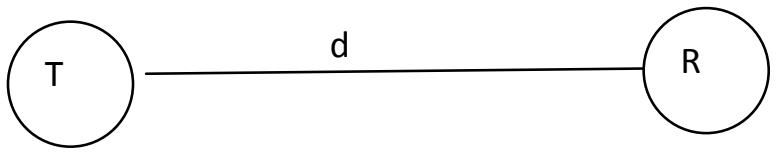

Figure.4a. Link connecting two nodes without relay nodes.

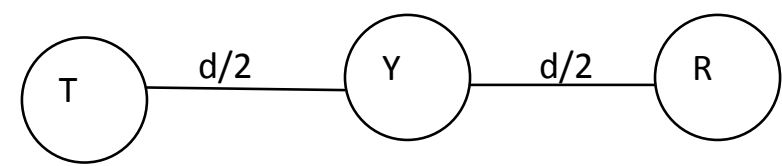

Figure.4b. Link connecting two nodes with a relay node

$\begin{array}{ll}P_{t}=P_{r}\left[(4 \pi d)^{2} L\right] /\left[G_{t} G_{r} \lambda^{2}\right] & 1.1 \\ P_{t}^{\prime}=P_{r y}\left[(4 \pi d / 2)^{2} L\right] /\left[G_{t} G_{r} \lambda^{2}\right] & 1.2 \\ P_{t y}=P_{r}^{\prime}\left[(4 \pi d / 2)^{2} L\right] /\left[G_{t} G_{r} \lambda^{2}\right] & 1.3\end{array}$

If $P_{r}=P_{r y}=P_{r}^{\prime}$ then $P_{t r}=P_{t}^{\prime}+P_{t y}$

$P_{t}>P_{t r}$ and $P_{t}=(n+1) * P_{t r}$ where $n$ is number of relay nodes.

$P_{t}=$ Transmit power without relay node; $P_{t r}=$ Total transmit power with relay node; $P_{t}{ }_{t}=$ Transmit power of first segment; $P_{t y}=$ Transmit power of second segment; $P_{r}=P_{r y}=P^{\prime}{ }_{r}=$ receive power. All other parameters are assumed to be same.

Further, $\boldsymbol{N}$ the number of relay nodes, which can lead to minimize energy consumption is optimized. Hence energy saving can lead to larger number of nodes/edges in the network compared to original network. This is in contrast to general topology control algorithms which mainly focus on reducing number of edges in order optimize energy consumption. However, the resulting super-graph must preserve connectivity of original nodes. The resulting topology can for instance be required; i) to maintain connectivity of the given nodes, ii) to be spanner of the underlying graph (the shortest path connecting a pair of nodes $u, v$ on the resulting topology is longer by a constant factor only than the shortest path between $u$ and $v$ on the given network), iii) to be plannar (no two edges in the resulting graph intersect). The objective must be to find a topology which meets one or a combination of such requirements.
In this paper, the focus is on the optimal transmission power of nodes by installing relay nodes to maintain the network connectivity. The goal of this research is to maximize the network lifetime by reducing transmit power at each node. In this work, first a scheme is presented to computing relay nodes required and their locations for a given transmission power, and the scheme must ensure the connectivity of network. Then, it is further modified to eliminate redundant edges in order to minimize interference. This algorithm is compared the algorithm with other popular algorithms in respect of MinMax and MinTotal. This algorithm is called as Power-Sensor (PS) algorithm. As shown in experiment results, the Power-sensor algorithm has good stability of network and promotes the energy-efficiency.

The remainder of this paper is organized as follows: In Section II presents related work with a focus on topology control and transmission power control in Wireless ad-hoc or sensor networks. In Section III presents a scheme for calculating the additional nodes and their locations for a given transmission power of nodes to sustain connectivity. The analysis and experimental results of the proposed algorithm are given in Section IV. Finally, concluded this paper in Section V with a summary of the work done and an outlook on future work.

Definition 1: MinMax: Maximum power that needs to be transmitted by any node to make network connected.

Definition 2: MinTotal: Minimum of total power transmitted by all nodes together in optimized connected network.

Definition 3:InterAvg: Average number of nodes that interfere per edge in the connected network.

Definition 4:InterMax: Maximum number nodes that can interfere to any edges in the connected network.

Definition 5: Network life time: Time elapsed before any node discharges its battery energy to a level which is not sufficient to transmit to its first-hop neighbor.

\section{RELATED WORK}

Many previous studies focused on solving topology control problems. Primarily, the algorithms focused on reducing number of edges to reduce energy consumption. Relative Neighborhood Graph (RNG) used to reduce the number of links between a node and its neighbors [1]. An edge belongs to the RNG only if it is not the longest leg of any triangle it may form in the original graph. N.Li [2] proposed a minimum Spanning Tree based algorithm for topology control. LMST is a localized algorithm to construct MST based topology in adhoc networks by using only information of nodes which are one hop away.

In recent years some new approaches have been proposed. In [3] the authors modeled the interaction among nodes as a game and analyzed the problem as non-cooperative game. In [4] authors proposed an algorithm to optimize the traditional topology control scheme. In this algorithm, each node iteratively increases its transmit power. In [5] Kenji proposed LTRT (Local Tree based Reliable Topology) which is motivated by LMST and TRT (Tree based Reliable Topology). LTRT can achieve nearly optimal performance at lower computational cost. Renato [6] presented three missed integer programming formulations for the $\mathrm{k}$-connected minimum consumption problem. Rajan [7] presented a semianalytical approach to analyze topological and energy related properties of K-connected MANETs. In [8] authors have analyzed the optimal transmission power of nodes according the optimal number of neighbors, and proposed the optimal topology control algorithm based on virtual clustering scheme. Authors in [9] analyzed the 
different approaches, constraints, and methods used for topology control algorithms.

Chen Wei et al [10] described an energy conservative unicast routing technique for multihop wireless sensor networks over Rayleigh fading channels. In Chen Wei model the assistant nodes transmissions can cause multiple packet reception at the receiving end and there by reordering requirement. In this model all the relay nodes are in-line so that they relay the same packet. So packets reach the destination in the same order. Jonathan et, al [11] focused on identifying the additional sensor placement for repairing and ensuring the fault-tolerance with $k$-connectivity. Present model is focusing more on reducing transmit power and thereby improving network life time while retaining connectivity. Martin [12] had presented a model identifying potential interference sources computing minimal interference path. To the best of our knowledge, all currently known topology control algorithms constructing only symmetric connections have in common that every node establishes a symmetric connection to at least its nearest neighbor. In other words all these topologies contain the nearest neighbor Forest [12] constructed on the given network. The symmetric connectivity is made with configuring the neighbors to appropriate transmit power level. In other words to preserve the connectivity, transmit power of the neighbors are adjusted to optimal level. However, in our model, we kept the transmit power of all nodes at lowest level possible and connectivity is preserved with adding relay nodes to compensate transmission distance. With this it is shown that inspite of increased number of nodes, transmit power on each edge is optimized.

\section{NETWORK MODEL}

In this paper multi-hop wireless network is considered, and assumed that each node able to gather its own location information via GPS or several localization techniques for wireless networks [13][14]. It represents a network as an undirected graph $\boldsymbol{G}=(\boldsymbol{V}, \boldsymbol{E})$ where $\boldsymbol{V}=\left\{\boldsymbol{v}_{\boldsymbol{l}}, \boldsymbol{v}_{2}, . ., v_{n}\right\}$ is a set of nodes randomly deployed in a two-dimensional plane. Each node $v \in V$ has a unique id, $\left(v_{i}\right)=i$ where $\boldsymbol{l} \leq \boldsymbol{i} \leq \boldsymbol{n}$ and is specified by its location. $\boldsymbol{E}$ is set of edges. Let $P_{i}=$ $\left[p_{\mathrm{i}}{ }^{1}, p_{\mathrm{i}}{ }^{2}, \ldots, p_{\mathrm{i}}{ }^{\mathrm{m}}\right]$ be a finite list of increasing power levels that can be assigned to node $\boldsymbol{i} \in \boldsymbol{V}$. It denotes $p_{\mathrm{i}}{ }^{1}$ the minimum power $p_{i}$ such that transmission from node $i$ reach at least one node in $\left.V_{i} i\right\}$. Further, $p_{\mathrm{i}}^{1+1}>p_{\mathrm{i}}^{1}$ for any $1=1, \ldots, m-1$. It defines $S_{\mathrm{i}}^{1}$ as the set of nodes reachable from node $i$ with the power assignment $p_{\mathrm{i}}=p_{\mathrm{i}}^{1}$ for any $l=1, . . m$. It may be noted that that $\bigcup_{l=1}^{l=m} S_{i}=V \backslash\{i\}$. For ease of notation, it is defined that $S_{0}=\phi$.

Initially all the nodes are transmitting with maximum power and are equipped with Omni directional antenna. It is assumed that each node can control the power of transmission to save energy consumption. Let $\boldsymbol{p}\left(\boldsymbol{v}_{i}, \boldsymbol{v}_{j}\right)$ be the power needed to support communication from node $\boldsymbol{v}_{\boldsymbol{i}}$ to $\boldsymbol{v}_{\boldsymbol{j}}$, and it is called symmetric if $p\left(v_{i}, v_{j}\right)=p\left(v_{j}, v_{i}\right)$. The power requirement is called Euclidean if it depends on the Euclidean distance $\boldsymbol{d}\left(\boldsymbol{v}_{\boldsymbol{i}}, \boldsymbol{v}_{\boldsymbol{j}}\right)$ [15]. Assuming unit disk model (UDG) maximum power a node can transmit is equal to the longest Euclidean distance among all pairs of nodes. For simplicity purpose the Euclidean distance of every pair of node is normalized with longest Euclidean distance. By topology control it can be shown that sub graph $\boldsymbol{G}^{\prime}=\left(\boldsymbol{V}, \boldsymbol{E}^{\prime}\right)$ of $\boldsymbol{G}$, in $\boldsymbol{G}$ ' the node has shorter and fewer numbers of edges as compare to $\boldsymbol{G}$. Power consumed by $\boldsymbol{G}, \leq \boldsymbol{G}$ is implied. To compute the subgraph, it starts with configuring all the nodes at lowest transmit power level. With that it computes the edges that are within communication distance. In addition, it also validates the edge as per the algorithms given below. Then it verifies if the subgraph is a connected network. Incase the subgraph is not connected network, it raises the transmit power of the nodes that are not connected to next level. It repeats the process till the subgraph is a connected network. Here with this model, it computes subgraph using different popular algorithms like GG, RNG, LMST, OTC, OTTC, XTC, and FLSS. For the subgraphs produced by each algorithm, it computes MinMax, MinTotal, number of edges, average interference of all edges (Intavg), Maximum interference on any edge (Intmax), and Average number of hops between two nodes.

Later, in this proposed algorithm, it assumes the nodes are configured initially at the lowest transmit power level possible $p_{\mathrm{i}}{ }^{1}, \mathrm{i}=1 \ldots \mathrm{N}$. At this power level we identify the edges that are within communication distance. Then in order to make the network connected, it identiies the unconnected edges and sort them in ascending order. It picks up each edge from sorted list and then compute number of relay nodes required to be installed between them and their locations so that the two nodes connected. Further, it also checks if the subgraph produced after adding relay nodes can give a connected network of original nodes. In case of not producing connected network, it goes to next edge from the list and repeat the process till a connected subgraph is produced.

Now it turns our attention to identify redundant nodes among the newly added relay nodes and remove them. For this purpose, it follows the greedy approach wherein it selects one node at a time and removes it. If the subgraph is still a connected network of original nodes, the edge is declared redundant and removed; otherwise it will be added back. It continues this for all newly added relay nodes and there by producing a connected subgraph with optimal number of additional nodes. Interference for an edge is defined [12] as $\operatorname{Cov}(e)=\mid\{w \in V \mid w$ is covered by $d(u,|u, v|)\} \bigcup\{w \in V \mid w$ is covered by $d(v,|v, u|)\} \mid$

\section{$\operatorname{InterMax}=\max \operatorname{Cov}(e) \in E$ and \\ InterAvg $x=\sum_{n=1}^{E} \operatorname{Cov}(e)$}

Theorem1: Any pair of unconnected wireless sensors can get connected by adding sufficient number of relays between the nodes at regular intervals without changing transmit power Proof of this is given through Lemma1 and Lemma2 below. Lemma1: Pair of nodes can be connected by adding $\frac{d(u, v)}{p_{u}}$ relays between the nodes.

Proof: Assuming omni-directional radio, power $p_{u}$ can communicate d. If $d(u, v)$ is more than $d, u \& v$ will not be able to communicate. However, by installing relay with $p_{u}$ at a distance $d$ from $u$ in the direction of $v$, we can extend the communication distance to $2 d$ distance. Thus by adding $\frac{d(u, v)}{p_{u}}$ we extend the communication distance upto $v$.

Lemma2: Additional relay do not disturb the existing connectivity. 
Proof: if $(w, v) \mid w \in V \leq p_{u}$ then if $w$ is in the direction $(u, v)$ then $d(u, w)+d(w, v)=d(u, v)$

Theorem2: Transmit power $P_{t}$ can be reduced by a factor of $n+1$ with $n$ relay nodes where $n>0$ to cover the communication distance.

Proof of this is given through Lemma3 and Lemma4 below. Lemma3: for free space communication, if distance $d$ between transmitter and receiver is reduced to $\frac{d}{k}$ then $P_{t}$ is reduced by $P, / k^{2}$

Proof: Let us start with the familiar free space communication equation $P_{r}=\left[P_{t} G_{t} G_{r} \lambda^{2}\right] /\left[(4 \pi d)^{2} L\right] \quad$ where $P_{r}$ is receive power, $P_{t}$ is transmit power and $d$ is distance between transmitter and receiver. And it can observed that $P_{t}$ is directly proportional to $d^{2}$. Hence by reducing the $d$ by $k$ times, required $P t$ gets reduced by $k^{2}$.

Lemma4: In free space communication total transmit power required by $k$ segments of equal distance is $k * P_{t}$.

Proof: Let us assume distance $d$ is divided in to $k$ equal segments. Relay node is placed at each segment. Transmit power required for each segment is $P_{t} / k^{2}$. Total transmit power required by $k$ segments is $P_{t} / k$.

\section{SIMULATION}

In order to demonstrate the effectiveness of this proposed algorithm, the Power-sensor algorithm is evaluated via extensive simulations and compared with other existing algorithms. Computational experiments have been carried out on a set of moderately sized network $(20,40,80,100,150,200$ nodes) with symmetric links MATLAB software as well as NS-2 simulator.

In the first experiment was done with 20 nodes distributed in $1000 \times 1000$ grid.

netXloc $=950.1293231 .1385606 .8426 \quad 485.9825$

$891.2990 \quad 762.0968 \quad 456.4677 \quad 18.5036 \quad 821.4072 \quad 444.7034$

$\begin{array}{llllll}615.4323 & 791.9370 & 921.8130 & 738.2072 & 176.2661 & 405.7062\end{array}$

$935.4697916 .9044 \quad 410.2702 \quad 893.6495$

netYloc $=57.8913 \quad 352.8681 \quad 813.1665 \quad 9.8613 \quad 138.8909$

$\begin{array}{lllllll}202.7652 & 198.7217 & 603.7925 & 272.1879 & 198.8143 & 15.2739\end{array}$

$746.7857445 .0964931 .8146465 .9943418 .6495 \quad 846.2214$ 525.1525202 .6474672 .1375

A connected network of the above nodes was generated using PS, RNG, GG, LMST, OTC, OTTC, XTC, and FLSS. The algorithms have been studied w.r.t MinToal, MinMax, InterAvg, and InterMax and results are plotted. Better performance of the proposed algorithm (PS) with respect to other algorithms is shown in figure6, figure7, figure8, figure9. It has also extended the study using NS-2 simulator. It has activated the energy model in NS-2 to capture the energy consumed by each node. Energy model computes energy consumed by each packet transmission and stores the residual energy at each node. It can run the simulation till any of the node residual energy becomes zero. This gives the network life time. It has simulated various sizes of the network with and without relay nodes. For RNG and GG algorithms, each node are configured to appropriate transmit power $P_{t}$. For PS algorithm, all nodes are configured at uniform $P_{t}$. The paper compared RNG and GG algorithms with PS algorithm. The consumed power includes energy consumed in transmitting the packet, receiving the packet, sensing power and idle power. This study used AODV as under lying routing protocol. The power consumed includes the impact of AODV overhead. For simplicity, the study has assumed power consumption for receiving packet, sensing power and idle power to be zero. Comparison of the network life time is plotted at figure13 which indicates increased life time for PS algorithm. MinMax is directly related to Network Life Time. It can be observed that number of relay nodes required decreases with increased density. So the gain in MinMax becomes negligible as the density increases as can be seen in figure7. This is obvious because the original nodes are so close that they can communicate with $P_{t}=p_{\mathrm{i}}{ }^{1}, \mathrm{i}=1 \ldots \mathrm{N}$ without relay nodes. Gain in MinMax or relay nodes impact is significant for sparsely deployed nodes. It has also computed the throughput and end-to-end delay for both cases and plotted the graphs at figure.11 \& figure.12. Increase in endto-end delay and decrease in throughput is implied due to increased hops in the communication. Since the objective is to reduce power consumption and increase in network life time, the variations in throughput and end-to-end delay are acceptable.

\section{CONCLSION}

As shown explained in the previous section, the proposed PS algorithm has clearly established improvement in MinMax, MinToatl, InterAvg and InterMax terms. However, cost of the relay nodes and increased end-to-end delay to be traded with the saving obtained in the above specified aspects. In the present study, the additional nodes are placed on Euclidean line to connect the unconnected nodes. However, further optimizations are also possible by position the additional nodes at optimal places.

\section{PS Algorithm}

Input: Set of $V$ nodes each $v \in V$ and each node is powered with lowest normalized power $p$

1. $G=(V, E)$ with all nodes configured with lowest normalized power $p . G$ is not connected graph

2. $G_{p s}=\left(V_{p s}, E_{p s}\right)$ is a connected graph with lowest normalized power $p$

3. $G=(V, E)$

4. $V_{p s}=\phi$

5. $E_{p s}=\phi$

6. $G_{p s}=\left(V_{p s}, E_{p s}\right)$

7. for all $v \in V$ do

8. $V_{p s}=V_{p s} \cup v$

9. end for

10. for all $e=(u, v) \in E$ do

11. if $v \neq u$ and $|(u, v)| \leq p$ and $|(v, u)| \leq p$ then

12. $E_{p s}=E_{p s} \bigcup\{e\}$

13. $E=E \backslash\{e\}$

14. end if

15. end for 
16. While $G_{p s}$ is not a connected graph do

17. $e=\min \{(u, v) \in E,|u, v|>p\}$

18. $N=\{(|u, v|-1) / p\}+1$

19. $\Delta N=|u, v| / N$

20. for $i=1$ to $N$

21. $u_{i}=$ location $u+\Delta N$ in $|u, v|$ direction

22. $\quad V p s \longleftarrow u_{i}$

23. $e=\left(u, u_{1}\right)$ or $\left(u_{i}, u_{i+1}\right)$ or $\left(u_{N}, v\right)$ as case may be

$$
\begin{aligned}
& \text { 24. } E_{p s}=E_{p s} \bigcup\{e\} \\
& \text { 25. } G_{p s}=\left(V_{p s}, E_{p s}\right)
\end{aligned}
$$

26. end for

27. $E=E \backslash\{e\}$

28. end while

29. while unprocessed $e=(u, v)$ where $\left(u \in V_{p s}\right.$ and $\left.\quad u \notin V\right) \quad$ or $\quad\left(v \in V_{p s}\right.$ and $v \notin V)$ or $(u, v \notin V)$ do

30. $G_{p s}^{\prime}=G_{p s} \backslash\{e\}$

31. if $G_{p s}^{\prime} \longrightarrow$ connected $G=(V, E)$ then

32. $G_{p s}=G_{p s}^{\prime}$

33. end if

34. end while

Output: $G_{p s}$ connected graph of $G$

\section{Figure-5 PS-Algorithm}
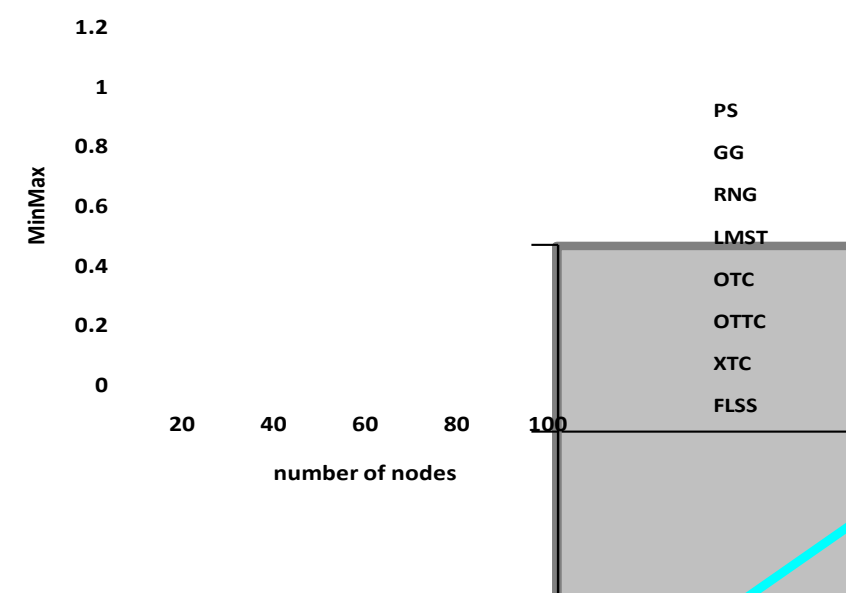

Figure.6a Comparison of MinMax for different algorithms

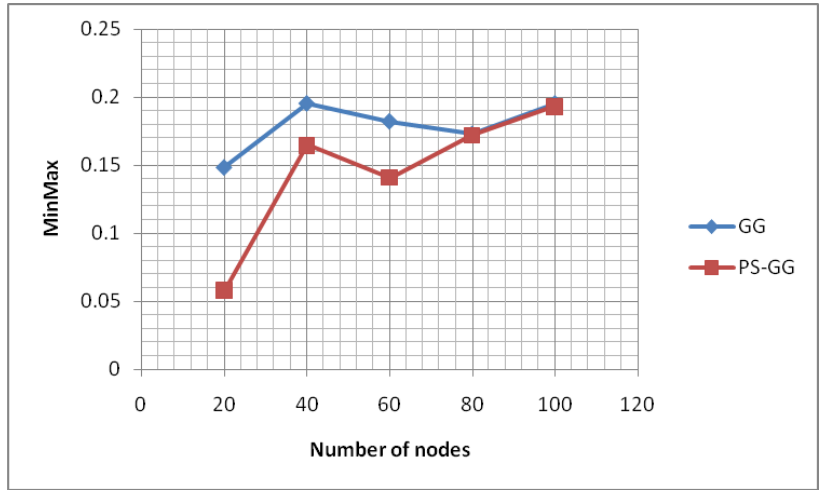

Figure 6b. Comparison of MinMax with and without relay nodes

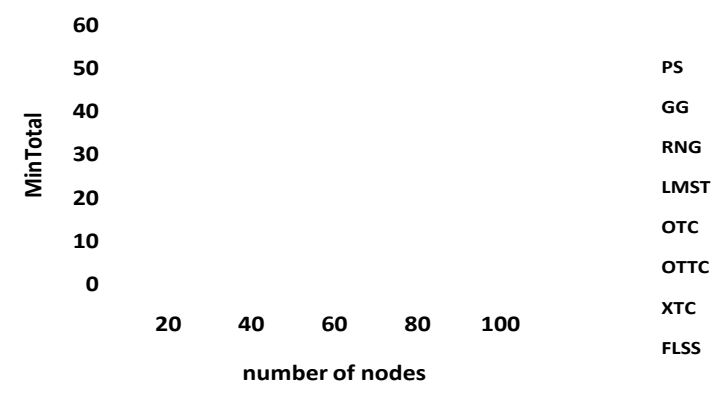

Figure7. Comparison of MinTotal for different algorithms

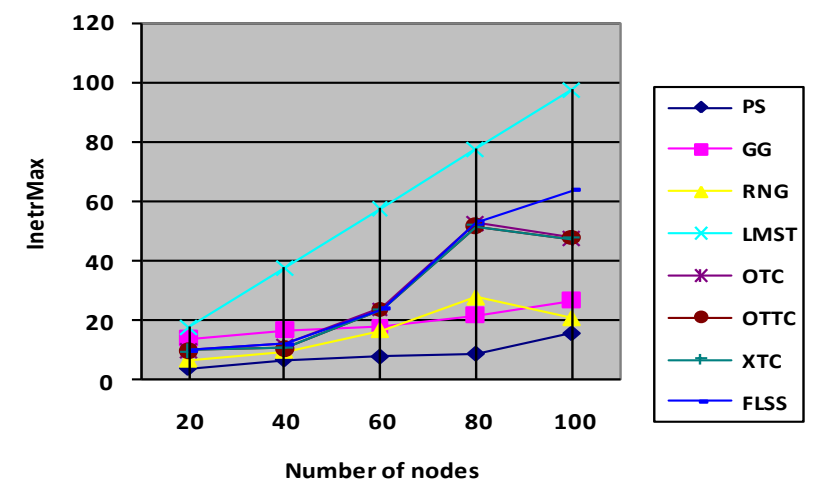

Figure.8 Comparison of InterMax for different algorithms 


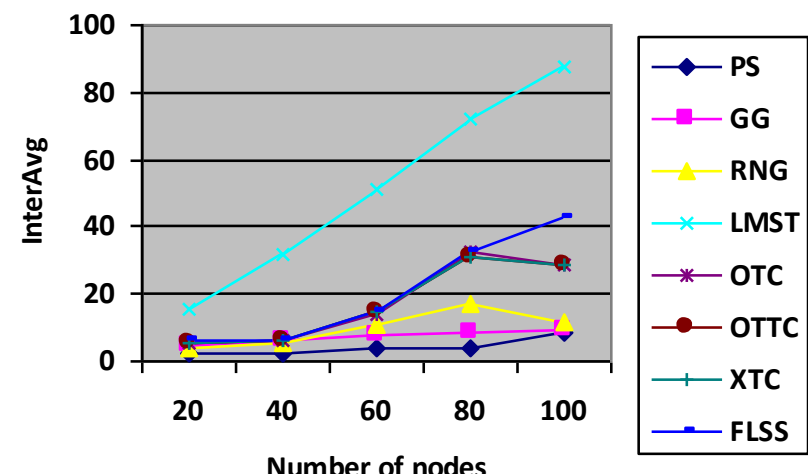

Figure9. Comparison of InterAvg for different algorithms

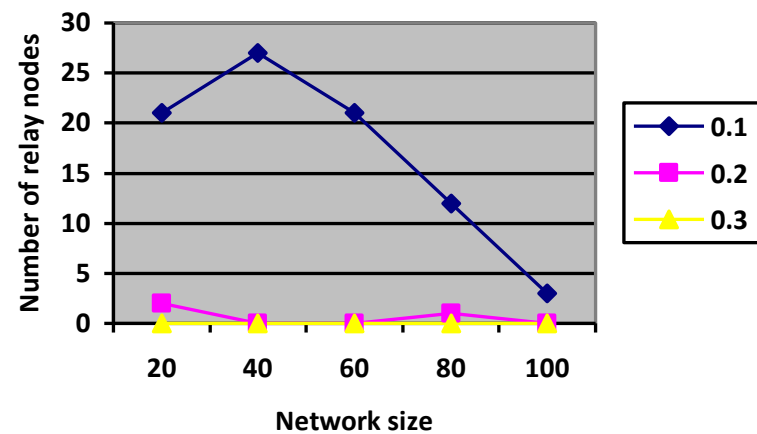

Figure 10. Number of relay nodes with different transmit power levels

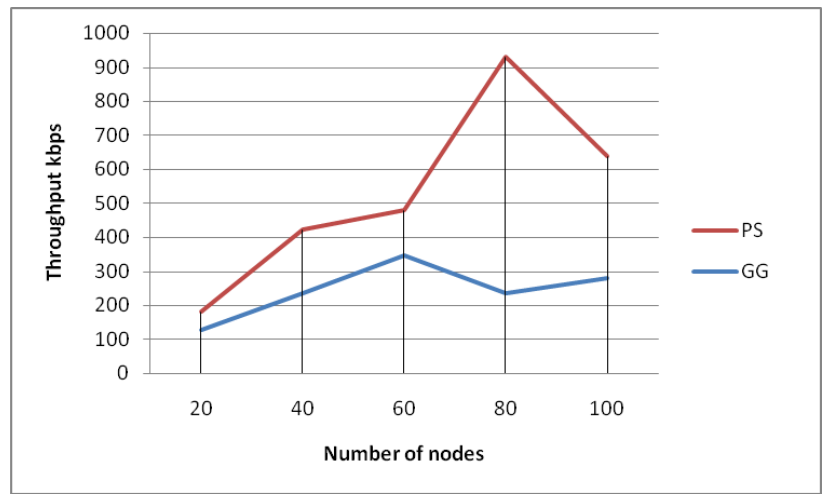

Figure 11. Comparison of throughput with and without relay nodes

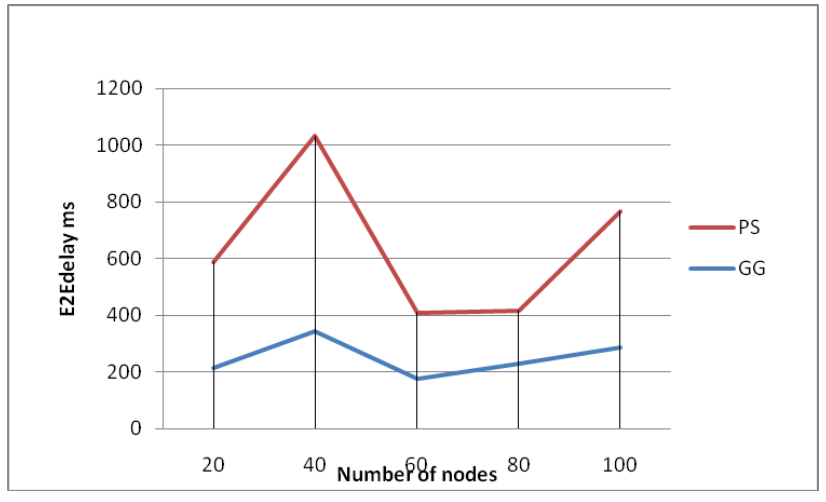

Figure 12. Comparison of end-2-end delay with and without relay nodes

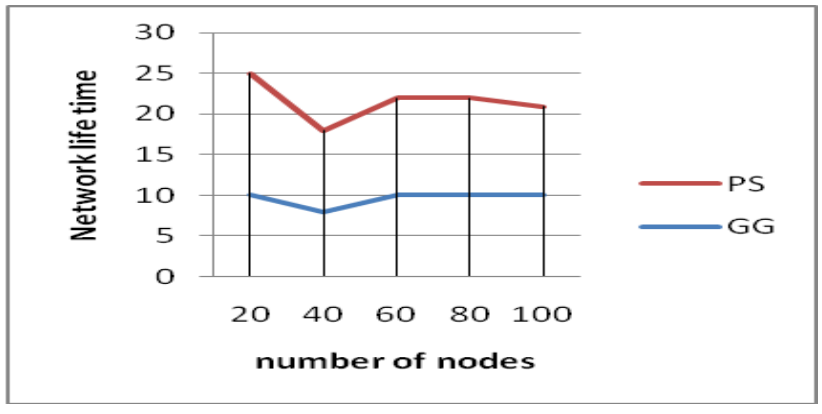

Figure 13 Network life time comparison with and without relay nodes

\section{REFERENCES}

[1] M. Cardei, J.Wu, and S.Yang, "Topology control in adhoc wireless networks using cooperative communication", IEEE Transactions on Mobile computing, vol.5, no.6, pp 711-724, 2006

[2] Kenji Miyao, Hidehia nakayama, Nirwan Ansari, and Nei Kato, "LTRT: An efficient and reliable topology control algorithm for ad-hoc networks", IEEE Transactions onwireless communications, vol.8, no.12, pp 6050-6058 Dec 2009

[3] Renato E.N.Moraes, Celso C. Ribeiro, and Christophe Duhamel, "Optimal Solutions for Fault-Tolerent Topology Control in Wireless Ad-hoc network", IEEE Transactions on wireless communications", vol.8, no.12, pp 5970-5981, Dec 2009

[4] M.A.Rajan , M.Girish Chandra , Lokanatha C. Reddy, and Prakash S. Hiremath, "Topological and Energy Analysis of $\mathrm{K}-$ Connected MANETs: A SemiAnalytical Approach", IJCSNS International Journal of Computer Science and Network Security, VOL.8 No.2, February pp199-206

[5] Xinhua Liu, Fangmin Li and Hailan Kuang, "An Optimal Power-controlled Topology Control for Wireless Sensor Networks", Proc. International Conference on Computer Science and Software Engineering, 2008, pp 550-554

[6] Niranjan Kumar Raya and Ashok Kumar Turuka, "Analysis of topology control alorithms in ad-hoc and sensor networks", Proc. International conference on challenges and applications of mathematics in science and technology (CAMIST), pp 562-571, Jan 2010

[7] Chen Wei et al, "AsOR: An Energy Efficient Multi-Hop Opportunistic Routing Protocol for Wireless Sensor Networks over Rayleigh Fading Channels", IEEE/ACM 
Transactions on wirless communications, vol-8, no-5, May, 2009

[8] Jonathan L.Bredin, Erik D.Demaine, Mohammad Taghi Hajiaghayi, and Daniela Rus, "Deploying Sensor Networks with guaranteed fault tolerance", IEEE/ACM Transactions on networking, vol-18,no-1, February 2010

[9] Martin Bhurkhart, Pascal von Rickenbanch, Roger Wattenhofer, Aaron Zollinger, "Does topology control reduce interference?" MobiHoc'04, May 24-26, 2004, Roppongi, Japan.

[10] G.Xing, C.Lu, Y.Zhang, Q.Huang, and R.Pless, "Minimum power configuration for wireless communication in sensor networks", ACM Transactions, Sensor networks, vol.3, pp200-233, 2007

[11] R.Madan and S.Lall, "” Distributed algorithms for maximum lifetime routing in wireless sensor networks", IEEE Transactions Wireless Communications vol.5 pp 2185-2193, 2006

[12] M.K.Maria and S.R.Das, "On-demand multipath distance vector routing in ad-hoc networks", Proceedings $9^{\text {th }}$ International Conference Network Protocols, Revierside, pp 14-23, 2001

[13] L.Lazos, andR.Poovendran, "SeRLoc: secure rangeindependent localization for wireless sensor networks",in Proc. ACM WiSe '04, 2004pp 21-30

[14] A. Caruso, S. Chessa, S.de, and A.Urpi, "GPS free coordinate assignment and routing in wireless sensor networks", Proc. IEEE INFOCOMM 2005, vol .1, pp 150-160 Mar 2005

[15] Ramanathan R and Redi J, "A Brief Overview of Ad Hoc Networks: Challenges and Directions," IEEE communication magazine, pp. 20-21, May 2002.

[16] L. Kirousis, E. Kranakis, D. Krizanc and A. Pelc, "Power Consumption in Packet Radio Networks", Theoretical Computer Science, pp. 289 - 305, 2000.

[17] G.Toussiant, The relative neighborhood graph of finite planar set, Pattern Recognition 12(4) (1980) 61-268

[18] K.R.Gabriel, R.R.Sokal, A new statistical approach to geographic variation analysis, Systematic zoology 18 (1969) 259-278

[19] Ning Li, Localized topology control in wireless networks, $\mathrm{PhD}$ thesis, 2005

[20] D.M.Blough, M.Leoncini, G.Resta, and P.Santi,"On the symmetric range assignment problem in wireless ad hoc networks", in Proc. $2^{\text {nd }}$ IFIP International conference onTCS, 2002, pp 71-82

[21] G.Calinescu, I.L.Mandoiu, and A.Zelikovsky, "Symmetricconnectivity with minimum power consumption in radio networks", Proc. $17^{\text {th }}$ IFIP World Comput. Congress, 2002 pp 119-130

[22] L.M.Kirousis, E.Kranakis,D.Krizanc, and A.Pelc, "Power consumption in packet radionetworks", Theoretical Computer Science, vol-243, no:1-2, 2000, pp 289-305
[23] V.kawadia, P.Kumar, "Power control and clustering in ad hoc networks", Proc of IEEE Infocom, San Francisco, CA, 2003, pp. 459-469

[24] L.Li, J.Y.Halpern, P.Bahl,Y.Wang, and R.Wattenhofer,"Analysis of a cone-based distributedtopology control algorithm for wireless multihop networks", in Proc. ACM PODC 2001,Aug. 2001, pp 264-273

[25] J. Cartigny, D. Simplot, and I.Stojmenovic, "Localized Minimum energy broadcasting in ad-hoc networks", in Proc. IEEE INFOCOMM 2003, vol.3, March 2003,pp 2210-2217

[26] N.Li, J.Hou, C.Sha,and L.Sha, "Design and analysis of anMST-based topology control algorithm", IEEE Transactions on wireless communications",vol.4, no.3, pp 1195-1206, May, 2005

[27] R.Komali, A.MacKenzie, and R.Gilles, "Effect of selfish node behavior on efficient topology design", IEEE Transactions on Mobile computing, vol.7, no.9, pp 10571070, sep 2008

[28] Kevin Chan , Ananthram Swami , Qing Zhao , and Anna Scaglione, "CONSENSUS ALGORITHMS OVER FADING CHANNELS “,The 2010 Military Communications Conference - Unclassified Program Netw orking Protocols and Performance Track

\section{AUTHORS PROFILE}

B.Brahma Reddy obtained his B.Tech from JNTU and M.Tech from IIT, Madras in 1980 \& 1982 respectively. He has worked for Indian Institute of Science, Indian Telephone Industries, National Informatics Centre, DishnetDSL, Reliance Infocomm for nearly 25 years. Past 6 years he is working as Professor in JNTU affiliated college. Currently he is perusing his Doctoral programme.

Prof. K.Kishan Rao obtained his B.E., M.E., degrees both in Distinction from O.U.College of Engineering , Osmania University in 1965 and 1967 respectively and Ph.D. from IIT Kanpur in the year 1973. Joined Regional Engineering College ,Warangal in 1972 and Retired as Principal in 2002. He has Guided 3 Ph.D. Candidates, 76 M.Tech. Dissertations ,5 Research Projects and published about 68 Technical Papers in National and international Journals and Conferences. He is a Senior Member of IEEE, Life Member of IETE, Life Member of ISTE and Life Member of APSA. His Research Interest are Wireless \& Mobile Communications, Adaptive Digital Signal Processing and OFDMA Networks. Presently working as Director and Adviser to Vaagdevi Group of Technical Institutions, Warangal. 